WPSReview International on Sustainable

Housing and Urban Renewal (RI-SHUR)

\title{
LAS EDUSI EN EL CONTEXTO DE LAS POLÍTICAS DE REGENERACIÓN URBANA EN ESPAÑA (1994-2018)
}

\author{
Sonia De Gregorio Hurtado \\ Departamento de Urbanística y Ordenación del Territorio \\ Universidad Politécnica de Madrid \\ Moneyba González Medina \\ Departamento de Ciencia Política y Relaciones Internacionales \\ Universidad Autónoma de Madrid
}

Artículo Recibido: 20/11/2017

Artículo Aceptado: 30/11/2017

\section{Resumen}

En los inicios de los 90, la actuación en la ciudad existente empezó a alejarse de la mera transformación física y a acercarse a un enfoque integrado que ponía en relación dicha transformación con la dimensión social, económica y medioambiental del desarrollo urbano. Esta evolución se manifestó en el ámbito comunitario con el lanzamiento de la Iniciativa Comunitaria URBAN (1994-1999), que promovió un método de regeneración urbana integrado basado en un enfoque multidimensional, estratégico y participativo. El mismo ejerció una influencia en el marco de la regeneración urbana de los Estados miembros que ha sido reconocida por la literatura. En el caso español, introdujo un modo diferente de abordar los problemas de degradación urbana que iba a tener continuidad en los programas URBAN II (20002006), la Iniciativa URBANA (2007-2013) y, en el periodo 2014-2020, en las Estrategias de Desarrollo Urbano Sostenible Integrado (EDUSI). Todos ellos mantienen el "enfoque URBAN", evidenciando su influencia sobre la acción política del Gobierno de España en materia de desarrollo urbano sostenible. Este trabajo hace una revisión del periodo 1994-2018 con el fin de identificar las influencias y relaciones entre el marco político y los instrumentos mencionados, para entender la evolución que ha dado lugar a las EDUSI y generar un conocimiento que pueda contribuir tanto al avance en el ámbito de la regeneración urbana como a la reflexión sobre la Agenda Urbana para España que el Ministerio de Fomento está desarrollando en la actualidad.

WPS RI-SHUR, n6, 2017, vol.2, ISSN: 2387-1768

MONOGRÁFICO SOBRE LAS ESTRATEGIAS DE DESARROLLO URBANO SOSTENIBLE E INTEGRADO (EDUSI) 


\title{
WPSReview International on Sustainable \\ Housing and Urban Renewal (RI-SHUR)
}

\begin{abstract}
In the early 1990s, the intervention on the existing city began to move away from mere physical transformation towards a more integrated approach, which put in relation this transformation with the social, economic and environmental dimensions of urban development. The launching of the URBAN Community Initiative (1994-1999) evidences this evolution at EU level, since it promoted an integrated urban regeneration method based on a multidimensional, strategic and participatory approach. The influence of URBAN-on the urban regeneration frameworks of the Member States has been recognized by the literature. In the Spanish case, it introduced a different way of addressing the problems of urban degradation that would continue in the URBAN II programmes (2000-2006), the URBANA Initiative (20072013) and, in during the period 2014-2020, in the so-called Integrated Sustainable Urban Development Strategies (EDUSI). This paper makes a review of the period 1994-2018 in order to identify the influences and relationships between the political framework and the aforementioned instruments, to understand the evolution that has given rise to the EDUSI. The analysis will deliver knowledge aimed to progress in the field of urban regeneration and to reflect about the Spanish Urban Agenda that the Ministry of Public Works is currently developing.
\end{abstract}

Palabras clave: Regeneración urbana, Estrategias de Desarrollo Urbano Sostenible Integrado-EDUSI, Política Urbana de la Unión Europea, enfoque URBAN

Keywords: Urban regeneration, Integrated Sustainable Urban Development Strategies -EDUSI, Urban Policy of the European Union, URBAN approach.

\section{Introducción}

En 1994, la Comisión Europea (CE) lanzó la Iniciativa Comunitaria URBAN en un contexto socioeconómico y ambiental que había llevado a un número amplio de actores a poner su atención sobre el declive de los barrios urbanos. Desde la segunda mitad de los años ochenta, las instituciones comunitarias ya habían empezado a dar forma a la idea de dedicar recursos económicos de los Fondos Estructurales para ayudar a las ciudades a afrontar sus problemas de degradación urbana. Esta reflexión se dio tanto en el seno de la CE como entre miembros del Parlamento Europeo (PE), derivando en el diseño de una Iniciativa Comunitaria dedicada de manera específica a

WPS RI-SHUR, n6, 2017, vol.2, ISSN: 2387-1768

MONOGRÁFICO SOBRE LAS ESTRATEGIAS DE DESARROLLO URBANO SOSTENIBLE E INTEGRADO (EDUSI) 


\section{WPSReview International on Sustainable \\ Housing and Urban Renewal (RI-SHUR)}

la regeneración urbana en el marco de la Política de Cohesión de la Unión Europea (UE) durante el periodo 1994-1999. A esta iniciativa se le dio el nombre de URBAN.

Su desarrollo en los diferentes marcos nacionales fue heterogéneo. Un aspecto que marcó claramente sus resultados fue la existencia (o no) de una política urbana de escala nacional en el Estado Miembro (EM). La contribución de URBAN fue mayor en aquellos países que no contaban con un marco nacional de política urbana (Carpenter, 2013), como era el caso de los países del Sur de Europa (España, Italia, Portugal, Grecia). La literatura que ha abordado la influencia de URBAN en los distintos marcos nacionales (Campagna, 2002; Chrorianopoulos, 2002; Tofarides, 2003; Carpenter, 2006; De Gregorio 2009, 2012a, 2014; Gutiérrez, 2010) ha puesto en evidencia que este instrumento ha hecho una contribución relevante en el marco de la regeneración urbana en los mismos.

En el caso español, URBAN llegó en un momento clave, cuando los Ayuntamientos democráticos salidos de las elecciones de 1979 ya habían desarrollado sus instrumentos de planeamiento (en particular, los Planes Generales de Ordenación Urbana), que guiarían su reforma interior y ordenarían su crecimiento. De este modo, al empezar los años noventa, casi todas las ciudades grandes y medias del país ya habían abordado este tema urgente, lo que permitía a los municipios poner su atención en otras cuestiones urbanas pendientes. Entre ellas aparecía como clave el tema de la degradación urbana, una cuestión que las ciudades habían empezado a abordar de manera sectorial y sin integrar todavía la cuestión central de la sostenibilidad.

En estos años, la emergencia del tema urbano en la agenda comunitaria coincidió con un conjunto de hitos importantes. En particular, la Conferencia sobre Medio Ambiente y Desarrollo de Naciones Unidas (Cumbre de la Tierra), celebrada en Río de Janeiro en 1992, en donde se alcanzó el consenso internacional para proponer a las ciudades el desarrollo de la denominada "Agenda 21 Local". Para avanzar hacia el desarrollo sostenible, las políticas urbanas debían asumir un enfoque que integrase la dimensión social, económica y ambiental, implicando a la comunidad local en dichas políticas. Este enfoque fue ratificado por la UE con la firma de la Carta de las Ciudades Europeas hacia la Sostenibilidad (Carta de Aalborg), adoptada en la Conferencia Europea sobre Ciudades Sostenibles en 1994, para prestar apoyo a las ciudades que

WPS RI-SHUR, n6, 2017, vol.2, ISSN: 2387-1768

MONOGRÁFICO SOBRE LAS ESTRATEGIAS DE DESARROLLO URBANO SOSTENIBLE E INTEGRADO (EDUSI) 


\section{WPSReview International on Sustainable \\ Housing and Urban Renewal (RI-SHUR)}

abordaran la elaboración e implementación de su Agenda 21 para la sostenibilidad urbana. De este modo, se va formalizando una nueva manera de orientar la acción en el medio urbano que resulta en una serie de instrumentos explícitos, entre los que se cuenta la mencionada Iniciativa Comunitaria URBAN.

URBAN proponía el desarrollo de programas de regeneración urbana caracterizados por un enfoque integrado (estratégico y participativo) ${ }^{1}$ en su metodología de diseño y actuación. En efecto, no solo se planteaba como un programa de regeneración urbana sino, sobre todo, como un método de trabajo ${ }^{2}$ para intervenir sobre los barrios en declive o desfavorecidos: "URBAN planteó su implementación a través de una metodología de trabajo específica. Caracterizada por asumir la multidimensionalidad de las dinámicas urbanas $y$, por lo tanto, la necesidad de integrar las acciones urbanísticas, sociales, económicas y medioambientales, pero también por el fomento de las prácticas innovadoras, [...] y la creación de redes de intercambio de conocimientos" (Gutiérrez, 2010: 163). La metodología de URBAN también promovía la innovación (basada en testar nuevas formas de solucionar los problemas), un enfoque estratégico basado en la consecución de objetivos, y un enfoque area-based que concentraba recursos económicos y técnicos, así como atención política en un área de actuación determinada en la que se trabajaba durante un periodo de unos siete años. Además, su filosofía pretendía dar lugar a una gobernanza multi-nivel colaborativa en el marco de la gestión de los programas de regeneración urbana (De Gregorio, 2012 y 2014a). En España, esta circunstancia introdujo una nueva forma de afrontar los problemas de la ciudad consolidada.

El grado de innovación que introdujo esta Iniciativa Comunitaria se desprende de la descripción de la práctica de la regeneración urbana recogida en el Primer Catálogo Español de Buenas Prácticas (MOPTMA, 1996). En particular, en la parte sobre Sostenibilidad y Políticas Urbanas y Locales, Fernando Prats señalaba que se estaba observando la formulación de "estrategias urbanas integradas (...) en las que se evidencia una sensibilidad generalizada en los poderes locales hacia la problemática urbanística y sociocultural de las ciudades". Sin embargo, también apuntaba que en ellas la percepción medioambiental todavía no superaba en muchos casos los aspectos físicos y paisajísticos más superficiales, "quedando relegados los aspectos

\footnotetext{
${ }^{1}$ Comunicación de la Comisión Europea a los Estados Miembros lanzando la Iniciativa Comunitaria URBAN (Diario Oficial $n^{\circ} \mathrm{L}$ 180, de 1/7/94. p 06-09).

${ }^{2}$ Que más tarde sería denominado "método URBAN" por la Comisión Europea (Comisión Europea, 2003).
}

WPS RI-SHUR, n6, 2017, vol.2, ISSN: 2387-1768

MONOGRÁFICO SOBRE LAS ESTRATEGIAS DE DESARROLLO URBANO SOSTENIBLE E INTEGRADO (EDUSI) 


\section{WPSReview International on Sustainable \\ Housing and Urban Renewal (RI-SHUR)}

más sustantivos relacionados con la sostenibilidad local'. Asimismo, añadía que la actuación local en esos años no tenía suficientemente en cuenta la sostenibilidad urbana y territorial, actuándose en base a "objetivos remediales, y prácticas segmentadas y cortoplacistas en el interior de las ciudades". El documento también mencionaba que se detectaba una "insuficiente innovación cultural e instrumental de los patrones de intervención en las ciudades" y señalaba como novedad que algunas ciudades habían empezado a desarrollar planes estratégicos, aunque casi todos tenían una visión muy sesgada hacia la mejora de la competitividad y la mejor inserción de las ciudades en las nuevas redes y dinámicas económicas a nivel nacional y europeo.

Por otra parte, es relevante señalar que en aquel momento la participación de la comunidad local en los procesos urbanos no era común. Los movimientos sociales y el asociacionismo habían perdido gran parte de su impulso de años anteriores, al quedar muchos de sus representantes de los mismos incorporados en los partidos democráticos presentes ahora en los plenos de los Ayuntamientos (Borja, 2001).

En este escenario, URBAN tenía un gran potencial de transformar la práctica de la regeneración urbana en el país. Algo que después se acentuó cuando la CE impulsó en 2000 la segunda fase de esta Iniciativa Comunitaria, que denominó URBAN II, y que se desarrollaría hasta 2006.

En el periodo 2007-2013 de la Política de Cohesión de la UE, URBAN llegó a su fin, ya que en el seno de la UE se decidió "transversalizar" la dimensión urbana de su política en los Programas Operativos de los EM, fundamentalmente a través del Fondo Europeo de Desarrollo Regional (FEDER). España fue uno de los pocos países que decidió dar continuidad a dicha experiencia a través de un programa que se denominó "Iniciativa URBANA", cuyo nombre recordaba inequívocamente al de la Iniciativa Comunitaria. En el contexto de la Política de Cohesión 2014-2020, se sigue dando continuidad al mismo a través del programa EDUSI (Estrategias de Desarrollo Urbano Sostenible Integrado).

El desarrollo de todos estos instrumentos, y la coherencia que existe entre ellos, hace de España un caso único en el escenario de la UE, al haberse dado en el marco de la dimensión urbana de la Política de Cohesión la continuidad del método URBAN

WPS RI-SHUR, n6, 2017, vol.2, ISSN: 2387-1768

MONOGRÁFICO SOBRE LAS ESTRATEGIAS DE DESARROLLO URBANO SOSTENIBLE E INTEGRADO (EDUSI) 


\section{WPSReview International on Sustainable \\ Housing and Urban Renewal (RI-SHUR)}

durante cuatro periodos de programación consecutivos: URBAN (1994-1999); URBAN II (2000-2006); Iniciativa URBANA (2007-2013); y EDUSI (2014-2020) (De Gregorio, 2017b). Esta circunstancia reviste mucho interés si se tiene en cuenta que España es un EM que nunca se ha dotado de una Política urbana nacional de carácter integrado, aun cuando en las últimas décadas ha asistido a la creciente importancia que el tema urbano ha ido adquiriendo en la política comunitaria.

En base a estos hechos, este trabajo profundiza en cada uno de estos instrumentos situándolos en el marco de la política de regeneración urbana en el que se ha desarrollado a nivel nacional. El objetivo es evidenciar la influencia mutua entre dicho marco de política y estos instrumentos, con el fin de generar conocimiento en torno a un tema poco abordado por la literatura e identificar resultados que puedan dar lugar a un conocimiento que potencie la eficacia de instrumentos futuros. Asimismo, se espera que el conocimiento generado por este trabajo pueda contribuir a la reflexión sobre la Agenda Urbana para España, que está siendo elaborada por el Ministerio de Fomento en el momento presente.

El trabajo se ha elaborado a partir de la investigación que las autoras vienen desarrollando sobre el tema en el marco de estudios previos. Al conocimiento que han acumulado se une un trabajo de revisión bibliográfica y de fuentes primarias, y un análisis de la información discursiva obtenida de entrevistas abiertas a autores clave así como de abundantes conversaciones informales con técnicos y académicos de distintos perfiles y con gran conocimiento del tema, tanto en el ámbito nacional como comunitario. La información obtenida se ha sistematizado en una línea temporal que coincide con los periodos de programación de la Política de Cohesión de la UE. La elección de esta estructura temporal ha venido derivada de la claridad con la que permitía abordar el tema, ya que los instrumentos en los que se pone el foco de este estudio han operado en el país en periodos coincidentes con los de la Política de Cohesión al estar co-financiados por el FEDER y el Fondo Social Europeo (FSE). Este criterio de organización del análisis ha permitido poner de manifiesto y profundizar en las relaciones e influencias entre el marco de política y los instrumentos, permitiendo alcanzar el objetivo del trabajo.

El trabajo se ha organizado de la manera siguiente: la parte IV aborda el periodo en el que se desarrolló URBAN (1994-1999), situando el instrumento en el marco de política

WPS RI-SHUR, n6, 2017, vol.2, ISSN: 2387-1768

MONOGRÁFICO SOBRE LAS ESTRATEGIAS DE DESARROLLO URBANO SOSTENIBLE E INTEGRADO (EDUSI) 


\title{
WPSReview International on Sustainable
}

\author{
Housing and Urban Renewal (RI-SHUR)
}

en el que se llevó a cabo. En las partes V, VI y VII, dedicadas a URBAN II, a la Iniciativa URBANA y a las EDUSI, respectivamente, se sigue el mismo criterio de análisis. Por último, la parte VIII se dedica al desarrollo de las conclusiones del estudio y a plantear algunos elementos de reflexión futura.

\section{URBAN y el marco nacional de polítca urbana en el periodo 1994-1999}

URBAN fue el primer instrumento de regeneración urbana de alcance nacional en el país. A su metodología novedosa, se unía la circunstancia de que el Gobierno de España (en adelante, Gobierno) no había puesto en marcha iniciativas de regeneración urbana a escala de país hasta ese momento, a diferencia de otros EM como Reino Unido o Francia. Desde los años 80, la principal política desarrollada en relación al tema urbano era la de vivienda que, aunque tenía un componente de mejora del ámbito donde se actuaba, nacía y se desarrollaba con un objetivo distinto, por lo que en este estudio no se considera como una experiencia relevante (hasta que en la década del 2000 se introduce en ella un enfoque orientado a la regeneración de manera más específica- ver apartado VI).

Es interesante, sin embargo, señalar que en este periodo el Gobierno estaba desarrollando un instrumento que abordaba el tema urbano a escala nacional. Esto se hacía desde las competencias de transporte en el medio urbano que ostentaba el Estado. Se trató del Plan Director de Infraestructuras 1993-2007 (PDI) aprobado en 1993, un instrumento de planificación para el desarrollo de las infraestructuras en el que se identificaban las necesidades del país y se afrontaban a través de proyectos de reactivación económica a escala regional financiados en parte con los Fondos Europeos. Desde el PDI se dio un intento por parte del Gobierno de ayudar a las ciudades y a las Comunidades Autónomas (CC.AA.) a desarrollar acciones de rehabilitación de gran escalas relacionadas con las infraestructuras de transporte, difíciles de acometer de manera unilateral por parte de los gobiernos locales y regionales. Además, con este instrumento se hizo un esfuerzo por hacer de la concertación multinivel un modo de actuar que pudiera trasladarse a otros ámbitos de política. Las acciones contenidas en el PDI se enmarcaron en cuatro líneas de actuación, siendo una de ellas la denominada "Política de ciudades". La problemática

WPS RI-SHUR, n6, 2017, vol.2, ISSN: 2387-1768

MONOGRÁFICO SOBRE LAS ESTRATEGIAS DE DESARROLLO URBANO SOSTENIBLE E INTEGRADO (EDUSI) 


\section{WPSReview International on Sustainable \\ Housing and Urban Renewal (RI-SHUR)}

urbana recibió atención desde la misma, constituyendo una de las principales aportaciones del instrumento, al ser la primera vez que un plan de ámbito estatal se dirigía específicamente al medio urbano, persiguiendo la rehabilitación e integrando acciones que generalmente se acometían de modo sectorial desde la política de infraestructuras y transporte.

La encargada de implementar la "Política de ciudades" del Ministerio de Obras Públicas, Transportes y Medio Ambiente (MOPTMA) fue la Dirección General de Actuaciones Concertadas en las Ciudades (ente que participaría en el lanzamiento y gestión de URBAN en España en sus primeros años de funcionamiento), dependiente de la Secretaría de Estado de Política Territorial y Obras Públicas. Entre sus funciones principales estaba la concertación con las CC.AA. y las Corporaciones Locales para efectuar actuaciones que integrasen la inversión en infraestructuras de transporte de competencia de la Administración General del Estado con los instrumentos propios del planeamiento urbano, que eran competencia de las CC.AA. y los Ayuntamientos.

EI PDI constituyó, por lo tanto, un esfuerzo valioso, a pesar de la sectorialidad que derivaba de su propia naturaleza de plan de infraestructuras a nivel estatal. Con el tiempo ganó reconocimiento porque supuso contar por primera vez con un instrumento que buscaba configurar un ámbito desde el que el Gobierno podía concertar con las CC.AA. y los Ayuntamientos actuaciones de importancia clave en el medio urbano. Sobre el PDI, Arce escribía en 1994: "se esboza una propuesta de realización de estudios y proyectos piloto, así como un Programa de Mejora del Medio Ambiente Urbano, con participación de diversos organismos implicados, que si se llevan a cabo, puede ser la oportunidad para iniciar una enriquecedora reflexión y el debate pendiente sobre nuestras ciudades, cómo actuar sobre su deterioro y la relación ciudad-desarrollo sostenible" (Arce, 1994).

Sin embargo, a partir de 1996 la Dirección General de Actuaciones Concertadas en las Ciudades desapareció de la estructura del MOPTMA como consecuencia del cambio político de ese mismo año. El nuevo Gobierno, dirigido por José María Aznar, además de renombrar al MOPTMA con el nombre de Ministerio de Fomento, llevó a cabo una

WPS RI-SHUR, n6, 2017, vol.2, ISSN: 2387-1768

MONOGRÁFICO SOBRE LAS ESTRATEGIAS DE DESARROLLO URBANO SOSTENIBLE E INTEGRADO (EDUSI) 


\section{WPSReview International on Sustainable \\ Housing and Urban Renewal (RI-SHUR)}

serie de cambios en su estructura. No consideró la labor que estaba llevando a cabo la Dirección General de Actuaciones Concertadas en las Ciudades dentro de sus objetivos, abandonando el enfoque concertado que había caracterizado al periodo anterior respecto a la acción en el medio urbano desde la competencia en infraestructuras de transporte del Ministerio (De Gregorio, 2012a). A partir de ese momento, los barrios degradados recibieron la atención del Estado principalmente a través de las políticas sectoriales de vivienda y de medio ambiente.

Entretanto, en 1994 había empezado a funcionar la Iniciativa Comunitaria URBAN, gestionada por la Dirección General de Fondos Comunitarios del Ministerio de Economía y Hacienda (MEH), con el apoyo de la Dirección General de Actuaciones Concertadas en las Ciudades del MOPTMA. La labor de la Dirección General de Actuaciones Concertadas en las Ciudades fue relevante en el proceso de preparación de programas presentados por las ciudades y su adecuación al "formato" URBAN, ya que se ocupó de ayudar a Ayuntamientos y CC.AA. a concebir programas basados en un enfoque integrado, en relación al cual no había tradición en el país. Entre sus funciones estuvo también el asesoramiento a las ciudades durante el proceso de implementación de los proyectos y la asistencia al Comité de Seguimiento de URBAN (De Gregorio, 2012a). Aun así, su función fue limitada. Por ejemplo, no pudo ejercer una función "correctora" sobre la metodología presentada por los programas en el ámbito de la participación de la comunidad local para que esta se asemejara más a lo que había quedado plasmado en la Comunicación de la Comisión (Comisión Europea, 1994) y en el resto de la documentación referente a la Iniciativa (entrevista a Félix Arias Goytre ${ }^{3}$ ). Tampoco consiguió ejercer una influencia relevante para dotar a URBAN de una visión de regeneración urbana multinivel, que integrase tanto a los tres niveles territoriales de gobierno como a otros actores relevantes (ibid.).

\footnotetext{
${ }^{3}$ Entrevista realizada en el marco de la Tesis Doctoral de Sonia De Gregorio (De Gregorio, 2012a).

WPS RI-SHUR, n6, 2017, vol.2, ISSN: 2387-1768

MONOGRÁFICO SOBRE LAS ESTRATEGIAS DE DESARROLLO URBANO SOSTENIBLE E INTEGRADO (EDUSI)
} 


\section{WPSReview International on Sustainable}

\section{Housing and Urban Renewal (RI-SHUR)}

URBAN se desarrolló en $31^{4}$ ciudades españolas. La novedad de su enfoque llevó a que los entes que gestionaron los programas (Ayuntamientos y CC.AA.) encontrasen problemas relevantes en su implementación. Los elementos metodológicos que las ciudades incorporaron en menor medida en sus estrategias fueron el enfoque integrado (entendido como multidimensionalidad o multisectorialidad de los proyectos y la creación de sinergias entre las diferentes acciones) y la participación (De Gregorio, 2012; 2014a). Las ciudades también encontraron limitaciones para adoptar un modo de gestionar los programas y justificar los gastos que les era ajeno (más cercano a la Nueva Gestión Pública y más alejado de la práctica administrativa tradicional) y que venía impuesto desde instancias comunitarias. En la superación de estas barreras se demostró clave la labor de la Dirección General de Fondos Comunitarios y el empeño de los entes gestores en sacar adelante sus programas. Estos últimos llegaron a su fin suponiendo una mejora de las áreas en las que actuaron. En muchos casos esa mejora no estuvo basada en la innovación y carácter demostrativo que se había fijado como objetivos URBAN (ibíd.). Sin embargo, desde una perspectiva general, URBAN inició un aprendizaje que iría calando poco a poco en los actores institucionales involucrados en el desarrollo de los programas, así como en los no institucionales, contribuyendo a dar visibilidad al tema de la regeneración urbana como marco de política pública en el país.

\section{URBAN II y el marco nacional de política urbana en el periodo 2000-2006}

En el periodo 2000-2006, desde el Gobierno se dio continuidad al enfoque de actuación en la ciudad existente asumido desde 1996. Una mirada general al mismo permite decir que se caracterizó por una falta de visibilidad del tema urbano en la acción del Ministerio de Fomento. Más allá del Plan de Vivienda 2002-2005, la actuación principal en las ciudades se dio dentro del Plan de Infraestructuras 2000-

\footnotetext{
${ }^{4}$ Programa Operativo URBAN 1994-1999: Badajoz, Badalona, Barakaldo, Cádiz, Cartagena, Huelva, La Coruña, Langreo, Madrid, Málaga, Sabadell, Salamanca, Sevilla, Toledo, Valencia, Valladolid y Vigo; Programa Operativo URBAN 1997-1999: Albacete, Avilés-Corvera, Castellón, Córdoba, León, Murcia, Palma de Mallorca, Pontevedra, Santa Coloma de Gramanet, Santander, Telde y Zaragoza. Dos de los programas de las Islas Canarias se desarrollaron dentro de la Iniciativa Comunitaria REGIS, por lo que de estos 31, 29 fueron programas URBAN y 2 programas REGIS implementados con la misma metodología y objetivos que URBAN.
}

WPS RI-SHUR, n6, 2017, vol.2, ISSN: 2387-1768

MONOGRÁFICO SOBRE LAS ESTRATEGIAS DE DESARROLLO URBANO SOSTENIBLE E INTEGRADO (EDUSI) 


\section{WPSReview International on Sustainable Housing and Urban Renewal (RI-SHUR)}

2007 que sucedió al PDI, pero asumiendo un enfoque marcadamente sectorial centrado en las infraestructuras urbanas de transporte (De Gregorio, 2012b).

En el año 2000 se lanzó la Iniciativa Comunitaria URBAN II. El número total de programas a financiar por el FEDER se redujo en ese periodo, lo que derivó en que en España sólo se llevasen a cabo 10 programas $^{5}$, un número muy inferior al del periodo anterior. Esto tuvo como consecuencia que en muchas CC.AA. no se desarrollara ninguno. En todo caso, la contribución de URBAN II al marco español fue relevante y más visible que la de URBAN I, aun cuando su dotación económica y número de programas fuera reducido. Por un lado, permitió mantener la atención sobre el tema de la regeneración urbana, y por otro, ejerció una influencia metodológica que se manifestó en otras iniciativas impulsadas por algunas CC.AA. como el programa de regeneración IZARTU en el País Vasco (2001) o la Ley de Barrios de Cataluña (2004). En la Comunicación de la CE por la que se lanzó URBAN II (Comisión Europea, 2000) se mantuvieron la misma metodología y objetivos generales que caracterizaron a su predecesor, aunque en este periodo se potenció el interés por la innovación y el intercambio de experiencias (sobre todo buenas prácticas) derivadas de los programas financiados (ibíd.).

Mientras URBAN II estaba en marcha, las Elecciones Generales de 2004 desembocaron en un cambio de color político en España que conllevó una mayor atención hacia el tema urbano. Desde el primer momento de la legislatura, el Gobierno de José Luis Rodríguez Zapatero otorgó una importancia explícita al mismo, que se manifestó principalmente en dos objetivos políticos: abordar los problemas del acceso a la vivienda y reorientar el modelo de desarrollo urbano. Desde este interés se empezaron a desarrollar un conjunto de acciones que derivarían en un conjunto relevante de estrategias y documentos políticos, en gran medida, bajo la coordinación y liderazgo del Ministerio de Medio Ambiente y Medio Rural y Marino (MARM), en la que también estuvieron presentes el Ministerio de Fomento y el recién creado

\footnotetext{
${ }^{5}$ Cáceres, Gijón, Granada, Jaén, Ourense, Pamplona, Sant Adrià de Besòs, San Cristóbal de La Laguna, San Sebastián-Pasaia y Teruel.
}

WPS RI-SHUR, n6, 2017, vol.2, ISSN: 2387-1768

MONOGRÁFICO SOBRE LAS ESTRATEGIAS DE DESARROLLO URBANO SOSTENIBLE E INTEGRADO (EDUSI) 


\section{WPSReview International on Sustainable \\ Housing and Urban Renewal (RI-SHUR)}

Ministerio de Vivienda (De Gregorio, 2012a; 2012b). Esta actividad se describe a continuación, como parte del periodo 2007-2013, ya que empezó a generar resultados concretos a lo largo del mismo.

\section{La Iniciativa URBANA y el marco nacional de política urbana en el periodo 2007-2013}

En este periodo concurrieron varias circunstancias, tanto a nivel nacional como comunitario, que condicionaron decisivamente el devenir de los hechos en relación al tema que nos ocupa. En 2007, el Gobierno publicó la Estrategia Española de Desarrollo Sostenible (MARM, 2007a). En ella se enmarcan un conjunto de documentos sucesivos de aplicación directa al medio urbano, en concreto: la Estrategia Española de Medio Ambiente Urbano (EEMAU) (MAMRM, 2008) y su documento programático, el Libro Verde del Medio Ambiente Urbano (MAMRM, 2007b), o la Estrategia Española de Sostenibilidad Urbana y Local (EESUL) (MAMRM, 2011). Además, en 2007 se aprobó la Ley del Suelo ${ }^{6}$, un paso clave para perseguir el cambio de modelo urbano al introducir el concepto de desarrollo urbano sostenible.

La EEMAU estableció, por primera vez, el modelo de ciudad y de desarrollo urbano recomendado para las ciudades españolas, señalando como objetivo proponer "las directrices y medidas que han de conducir a los pueblos y ciudades de España hacia escenarios más sostenibles manteniendo su competitividad" (MARM, 2007a: 6). Potenciaba el modelo de ciudad compacta, compleja, eficiente y cohesionada socialmente con la meta de mejorar la calidad urbana de los pueblos y ciudades y el aumento de la calidad de vida de la ciudadanía. El documento apostaba abiertamente por la regeneración urbana y la recuperación de brownfields en el interior de las ciudades, para "recuperar y rehabilitar la ciudad existente" (MAMRM, 2007:12). Proponía como una de sus directrices priorizar la recuperación y rehabilitación de la ciudad frente a la creación de nuevos sectores de expansión, absorbiendo el

\footnotetext{
${ }^{6}$ BOE núm. 128, de 29 de mayo de 2007.
}

WPS RI-SHUR, n6, 2017, vol.2, ISSN: 2387-1768

MONOGRÁFICO SOBRE LAS ESTRATEGIAS DE DESARROLLO URBANO SOSTENIBLE E INTEGRADO (EDUSI) 


\section{WPSReview International on Sustainable}

\section{Housing and Urban Renewal (RI-SHUR)}

crecimiento dentro de la misma, rehabilitando las zonas vulnerables y barrios en crisis social y ambiental, fomentando la integración social y la mezcla de usos y rentas en todas las áreas urbanas (MARM, 2007a). Las demás estrategias y documentos mencionados desarrollaron una visión consistente en la descrita en la EEMAU en materia de urbanismo y regeneración urbana. La visión recogida en la EEMAU estaba en línea con el discurso de las instituciones comunitarias en materia de desarrollo urbano, y tuvo su reflejo práctico en tres actuaciones relevantes desarrolladas desde el Gobierno en el periodo que nos ocupa: la definición del Área de Renovación Urbana (ARU) del Plan de Vivienda 2005-2008, el lanzamiento de la Iniciativa URBANA y los proyectos integrales de desarrollo local y urbano -PIDLU- en el año 2007.

Paralelamente, en el ámbito comunitario durante la Reunión Informal de ministros de desarrollo urbano de la UE que se celebró en Leipzig en 2007, se acordó la "Carta de Leipzig sobre Ciudades Europeas Sostenibles" ${ }^{7}$, un documento que "puede considerarse como la primera institucionalización en la Unión Europea del enfoque integrado en el desarrollo urbano sostenible" (Instituto Universitario de Urbanística, 2010: 36). La Carta influyó de manera notable en la evolución de la política urbana de la UE en la década siguiente y en la definición de la Iniciativa URBANA, el instrumento a través del que España optó por dar continuidad a URBAN. La penetración en España de la política de desarrollo urbano de la UE, inspirada en el "enfoque URBAN", se fue articulando en el contexto de la programación de los fondos de la Política de Cohesión de la UE (González, 2013). En primer lugar, a través del establecimiento de un eje dedicado al desarrollo urbano en la programación del FEDER (Eje de Desarrollo local y urbano), recogido en el Marco Estratégico Nacional de Referencia 2007-2013 del Estado español MENR. En segundo lugar, mediante la creación de un programa específico de desarrollo urbano inspirado en URBAN (Iniciativa URBANA). En tercer lugar, a través de los documentos que contenían las directrices metodológicas para la

\footnotetext{
${ }^{7}$ A raíz de la Carta de Leipzig, se produjo una mayor visibilidad de la cuestión del desarrollo urbano en la agenda política comunitaria. Junto a la publicación del Informe de expertos "Cities of Tomorrow" (2011), la Dirección General de Política Regional cambió de manera significativa su nombre por el de "Dirección General de Política Regional y Urbana" (2012), visibilizando la creciente importancia que el tema urbano estaba adquiriendo en el marco comunitario.
}

WPS RI-SHUR, n6, 2017, vol.2, ISSN: 2387-1768

MONOGRÁFICO SOBRE LAS ESTRATEGIAS DE DESARROLLO URBANO SOSTENIBLE E INTEGRADO (EDUSI) 


\section{WPSReview International on Sustainable \\ Housing and Urban Renewal (RI-SHUR)}

elaboración de las propuestas para las convocatorias: "Iniciativa URBANA (URBAN). Orientaciones para la elaboración de propuestas" (Ministerio de Economía y Hacienda, 2007b).

La Iniciativa URBANA se desarrolló a través de 46 programas en otras tantas ciudades, en las que se pusieron en marcha estrategias multisectoriales que abordaban cuestiones ambientales, sociales, económicas, turísticas, culturales, patrimoniales, nuevas tecnologías, sociedad de la información, etc. centradas en abordar la desventaja social y económica de un área urbana seleccionada. Las propuestas debían cumplir una serie de requisitos: 1) adoptar un enfoque integrado; 2) estar basadas en un diagnóstico sólido de la zona de intervención; 3) establecer una estrategia, metas y acciones definidas; 4) estar alineadas y ser coherentes con las políticas de la UE, así como con la Estrategia de Lisboa y la Estrategia de Gotemburgo; 5) llevar a cabo una descripción de los sistemas de control y gestión, y 6) establecer una estrategia basada en el principio de asociación (participación ciudadana y colaboración público-privada).

El programa jugó un papel muy importante en continuar el camino iniciado por URBAN, especialmente desde el punto de vista de la gobernanza local (Huete et al., 2016) y visibilizar más el tema de la regeneración a nivel nacional (De Gregorio 2017a). Su análisis ha permitido identificar que se han dado pasos relevantes sobre algunos aspectos que definen el "método URBAN", particularmente la coordinación dentro de los ayuntamientos con el fin de dar lugar a estrategias integradas al actuar de manera simultánea en diferentes ámbitos sectoriales. Sin embargo, dicho análisis también ha permitido poner a la luz cuestiones relacionadas con el enfoque integrado y la participación de la comunidad local en las que las ciudades no han conseguido avanzar mucho más de lo que se hizo en el contexto de URBAN II (De Gregorio 2017a y b). La Iniciativa URBANA estuvo reforzada por la Red de Iniciativas Urbanas (RIU), una de las redes sectoriales que preveía el Marco Estratégico Nacional de Referencia, y que nacía con los objetivos principales de ser un foro del intercambio de

WPS RI-SHUR, n6, 2017, vol.2, ISSN: 2387-1768

MONOGRÁFICO SOBRE LAS ESTRATEGIAS DE DESARROLLO URBANO

SOSTENIBLE E INTEGRADO (EDUSI) 


\section{WPSReview International on Sustainable}

\section{Housing and Urban Renewal (RI-SHUR)}

experiencias y difusión de buenas prácticas en regeneración urbana (Ministerio de Economía y Hacienda, 2007a: 223). La RIU ha jugado un papel muy relevante en relación a estos dos objetivos desde que fue creada en 2009, aunque lejos del potencial que presentaba y los objetivos que se puso inicialmente (De Gregorio, 2017b).

Para completar este marco, hay que recordar que en el primer semestre de 2010 tuvo lugar Presidencia Española ${ }^{8}$ de la UE. Durante la misma el Gobierno español mostró un interés específico en contribuir al avance de la reflexión sobre la cuestión urbana en el marco de la UE, asumiendo un papel activo en esta materia. Este interés hay que entenderlo en el contexto de la mayor atención que el tema urbano había alcanzado dentro de la acción política del Gobierno desde 2004 y que desembocó en la Declaración de Toledo (2010), acordada por Reunión Informal de ministros de desarrollo urbano de la UE. Una de las principales contribuciones de la misma fue aportar una propuesta de elementos clave "del enfoque integrado en la regeneración urbana en particular y en el desarrollo urbano en general' con el fin de avanzar hacia un entendimiento común de lo que dicho enfoque significaba en el marco europeo.

En los años finales de este periodo el Ministerio de Fomento y el Ministerio de Medio Ambiente siguieron impulsando diversas iniciativas centradas en avanzar hacia la sostenibilidad del desarrollo urbano. A ello contribuyó el protagonismo que este tema adquirió en la agenda política de la UE en ese momento y la oportunidad que supuso

\footnotetext{
${ }^{8}$ En el contexto de la preparación de la Presidencia Española de la UE, el Ministerio de Fomento encargó al Instituto Universitario de Urbanística de Valladolid la elaboración de un informe sobre las políticas de regeneración urbana integrada en la UE, que procurase "una visión panorámica sobre cómo se está enfocando en los diversos países de la UE la "regeneración urbana integrada", entendida ésta como una estrategia fundamental para alcanzar, entre otros, los objetivos señalados en la Carta de Leipzig". A la luz de este trabajo, se pone de manifiesto que el concepto de regeneración urbana se relacionaba principalmente con la degradación física de los edificios, seguida de la concentración de problemas sociales y de la demanda de nuevos espacios públicos y equipamientos en los distintos Estados miembros (Instituto Universitario de Urbanística, 2010: 24). Es de interés señalar que las principales conclusiones que arrojaba el informe para el caso español indicaban que, a diferencia de otros marcos nacionales, en el país no se había desarrollado una política nacional de regeneración urbana/desarrollo urbano de carácter específico, considerándose que la atención al tema urbano por parte del Gobierno seguía concentrándose en las políticas de vivienda y de patrimonio cultural (Instituto Universitario de Urbanística, 2010).
} 


\section{WPSReview International on Sustainable \\ Housing and Urban Renewal (RI-SHUR)}

la Presidencia Española de la UE. Además de los documentos señalados más arriba, entre estas iniciativas "domésticas" destacan la creación del Sistema de Información Urbana $^{9}$ (SIU), el Observatorio de la Vulnerabilidad Urbana ${ }^{10}$ y el "Sistema de Indicadores de Sostenibilidad Local", que respondían a la necesidad de obtener información sistematizada y datos concretos sobre la realidad urbana española. Sin embargo, el Gobierno no lanzó instrumentos específicos de regeneración urbana, como sí había pasado en Italia y Portugal, en gran medida, debido a la influencia de la política urbana de la UE y en particular de URBAN (De Gregorio et al., 2013). De esta manera la Política de Cohesión de la UE siguió siendo el marco que "suplía" la falta de apoyo específico a la regeneración de los barrios degradados de las ciudades desde el nivel del Gobierno

En contrapartida a esto, hay que recordar que España el único de los países que habían implementado URBAN, dio continuidad a su experiencia a través de un instrumento específico. A la luz de lo mencionado más arriba esto puede aparecer como una paradoja, que se entiende en base al marco competencial vigente en España en relación al tema urbano en virtud de la Constitución de 1978.

En 2011 se produjo un cambio de partido en el Gobierno de España, liderado por Mariano Rajoy. El mismo se distanció de la acción política de su antecesor en relación al tema urbano, reduciendo la atención hacia el mismo. Esto hizo que muchos de los instrumentos lanzados por el Ejecutivo anterior perdieran visibilidad. De manera interesante, esta situación cambió en 2013, cuando en abril el Gobierno aprobó el Plan estatal de fomento del alquiler de viviendas, la rehabilitación edificatoria, y la regeneración y renovación urbanas 2013-2016, y en junio CAMBIAR A ARIAL 11 la Ley 8/2013 de Rehabilitación, Regeneración y Rehabilitación de Áreas Urbanas ${ }^{11}$. Ambos instrumentos constituían un nuevo marco jurídico a través del que se perseguía potenciar la vivienda en alquiler y continuar el cambio de paradigma en relación al

\footnotetext{
${ }^{9}$ https://www.fomento.gob.es/MFOM/LANG_CASTELLANO/DIRECCIONES_GENERALES/ARQ_VIVIEND A/SUELO_Y_POLITICAS/SIU/

10 http://www. fomento.es/MFOM/LANG CASTELLANO/DIRECCIONES GENERALES/ARQ VIVIENDA/S UELO Y POLITICAS/OBSERVATORIO/BOE núm.153, de 27 de junio de 2013
}

WPS RI-SHUR, n6, 2017, vol.2, ISSN: 2387-1768

MONOGRÁFICO SOBRE LAS ESTRATEGIAS DE DESARROLLO URBANO

SOSTENIBLE E INTEGRADO (EDUSI) 


\section{WPSReview International on Sustainable \\ Housing and Urban Renewal (RI-SHUR)}

desarrollo urbano que había iniciado la Ley del Suelo de 2007. Su potencial empezaría a desplegarse en el periodo sucesivo, situándose en el caso de la Ley 8/2013 en el interés en potenciar la colaboración público-privada en la rehabilitación, la regeneración y la rehabilitación.

\section{Las EDUSI y el marco nacional de política urbana en el periodo 2014-2020}

La progresiva "explicitación" de la política urbana de la UE tuvo su correlato en la Política de Cohesión para el periodo 2014-2020, especialmente a través de dos hitos importantes. Por un lado, la obligatoriedad de que al menos un $5 \%$ de los recursos del FEDER se destinase a financiar estrategias integradas de desarrollo urbano sostenible (artículo 7 del Reglamento FEDER ${ }^{12}$ ); por otro, la re-dinamización del proceso de elaboración de una "Agenda urbana para la Unión Europea", que conduciría a la aprobación del Pacto de Ámsterdam, en la Reunión Informal de Ministros de Desarrollo Urbano, celebrada el 30 de mayo de 2016.

La continuidad del "enfoque URBAN", y la importancia del FEDER en este proceso, habían sido reconocidas en el informe "Cities of Tomorrow" (2011). Como se ha venido señalando, la singularidad del enfoque radica en su metodología, que se concreta en una serie de requisitos procedimentales dirigidos a garantizar la aplicación del enfoque integrado (estratégico y participativo). Dicha metodología, compleja y muy distinta de la gestión ordinaria de las Administraciones locales españolas, exige un gran "esfuerzo pedagógico ${ }^{13}$ y técnico por parte de las instituciones y una inversión en recursos humanos especializados que puedan encargarse del diseño y la implementación de los proyectos.

Del mismo modo que había ocurrido en el periodo anterior, España dio continuidad ${ }^{14}$ al enfoque URBAN, en primer lugar, a través del establecimiento de un eje

${ }^{12}$ Reglamento (UE) no $1301 / 2013$.

${ }^{13}$ Una evidencia de este condicionamiento se puede observar en el siguiente titular: "La UE marca las directrices a los concellos para que ejecuten los fondos Edusi" [La Voz de Galicia. 09/09/2017. Enlace:https://www.lavozdegalicia.es/noticia/coruna/arteixo/2017/09/09/ue-marca-directrices-concellosejecuten-br-fondos-edusi/00031504974827670587347.htm]

${ }^{14}$ A esta continuidad hace mención expresa el Considerando 4 de la Orden HAP/2427/2015, de 13 de noviembre, por la que se aprueban las bases y la primera convocatoria para la selección de estrategias de Desarrollo Urbano Sostenible e Integrado que serán cofinanciadas mediante el programa operativo FEDER de crecimiento sostenible 2014-2020, al señalar "La voluntad de dar continuidad a la provechosa 


\section{WPSReview International on Sustainable}

\section{Housing and Urban Renewal (RI-SHUR)}

específicamente dedicado al desarrollo urbano dentro del Programa Operativo de Crecimiento Sostenible ${ }^{15}$ del FEDER (Eje 12: Desarrollo urbano integrado y sostenible $\left.{ }^{16}\right)$, en virtud de lo establecido en el "Acuerdo de Asociación de España para el periodo 2014-2020", aprobado el 30 de octubre de 2014. En segundo lugar, mediante la creación de un programa específico de desarrollo urbano denominado "Estrategias de Desarrollo Urbano Sostenible Integrado" (EDUSI 2014-2020). En tercer lugar, a través de las directrices metodológicas para la elaboración de las estrategias, recogidas en el documento "Orientaciones para la definición de Estrategias de Desarrollo Urbano Sostenible Integrado en el periodo 2014-2020" (Red de Iniciativas Urbanas, 2015), en línea con el documento elaborado por la Comisión Europea en 2015 "Orientaciones para los Estados miembros Desarrollo sostenible integrado en el medio urbano Reglamento del FEDER“.

El programa EDUSI se dirige a municipios o áreas funcionales con una población de al menos 20.000 habitantes. La primera ${ }^{17}$ convocatoria fue publicada en el mes de noviembre de 2015, a la que siguieron otras dos, publicadas en octubre de $2016^{18} \mathrm{y}$ septiembre de 2017, respectivamente. Esta última se caracterizó por dar un mayor peso a la dimensión de inclusión social (OT 9), de acuerdo con las recomendaciones de la Comisión Europea. Las principales diferencias respecto a la Iniciativa URBANA se concretan en que se financian estrategias (no proyectos) y se introduce la posibilidad de que el ámbito de actuación sea un área funcional. En la orden de la primera convocatoria se describen los elementos de orden metodológico que ha de incluir la estrategia (art. 8.1). Estos son:

1) La identificación inicial de los problemas o retos urbanos del área, que constituyen un obstáculo para lograr un verdadero desarrollo urbano sostenible. Para ello, deben tenerse en cuenta los cinco retos del artículo 7

\footnotetext{
experiencia española derivada del desarrollo de la Iniciativa URBANA, la Iniciativa Comunitaria URBAN y los Proyectos Pilotos Urbanos, cuyos inicios se remontan a principios de los años 90".

${ }^{15}$ En el POCS, los retos urbanos se relacionan con los Objetivos Temáticos (OT) siguientes: OT2 Mejora del acceso, del uso y de la calidad de las tecnologías de la información y la comunicación; OT4 Apoyo de la transición hacia una economía de bajas emisiones de carbono (obligatorio); OT6 Preservación y protección del medio ambiente y fomento de la eficiencia de los recursos; y OT9 Fomento de la inclusión social y la lucha contra la pobreza y contra cualquier tipo de discriminación (obligatorio).

${ }^{16}$ Este Eje está dotado con 1.012.754.015,00 euros, según lo aprobado en el POCS del FEDER.

17 Se presentaron 256 propuestas EDUSI y fueron seleccionadas 83 (709.037.163,09 euros).

Enlace: https://www.boe.es/boe/dias/2016/12/14/pdfs/BOE-A-2016-11863.pdf

${ }_{18} \mathrm{Se}$ presentaron 185 propuestas EDUSI y fueron seleccionadas 40 (297.484.353 euros). Enlace: http://www.boe.es/boe/dias/2017/05/22/pdfs/BOE-A-2017-5678.pdf
} 


\section{WPSReview International on Sustainable \\ Housing and Urban Renewal (RI-SHUR)}

del Reglamento FEDER (económicos, ambientales, climáticos, demográficos y sociales)

2) Un análisis del conjunto del área urbana desde una perspectiva integrada, que abarque el ámbito físico, medioambiental y climático, energético, económico, demográfico, social, el contexto territorial, el marco competencial, los instrumentos de planificación y un análisis de riesgos.

3) Un diagnóstico de la situación del área urbana (por ejemplo, un análisis DAFO), así como la definición de los resultados esperados y su cuantificación: valor actual y valor objetivo al final del 31 de diciembre de 2022 en base a los indicadores de resultado definidos. También deben definirse las prioridades, a partir de una jerarquización de los retos y objetivos;

4) La delimitación del ámbito de actuación (que puede ser geográfica o funcional), además de la población afectada. Esta delimitación debe estar basada en indicadores y en una justificación de que la escala a la que se propone actuar es la adecuada para afrontar los retos identificados.

5) Un Plan de Implementación de la Estrategia, que incluirá los siguientes elementos: a) descripción de las líneas de actuación a llevar a cabo por cada Objetivo Temático; b) descripción, para cada línea de actuación, de los criterios y procedimientos para la selección de las operaciones; c) cronograma orientativo de las líneas de actuación; d) presupuesto indicativo del Plan de Implementación y e) indicadores de productividad.

6) La descripción de los mecanismos de participación ciudadana y de los agentes sociales;

7) La garantía de la capacidad administrativa para su implementación; y

8) Referencia a los principios horizontales y objetivos transversales: igualdad entre hombres y mujeres y no discriminación; desarrollo sostenible; accesibilidad; cambio demográfico; y mitigación y adaptación al cambio climático.

WPS RI-SHUR, n6, 2017, vol.2, ISSN: 2387-1768

MONOGRÁFICO SOBRE LAS ESTRATEGIAS DE DESARROLLO URBANO SOSTENIBLE E INTEGRADO (EDUSI) 


\section{WPSReview International on Sustainable}

\section{Housing and Urban Renewal (RI-SHUR)}

Figura 1. Directrices para la elaboración de una EDUSI

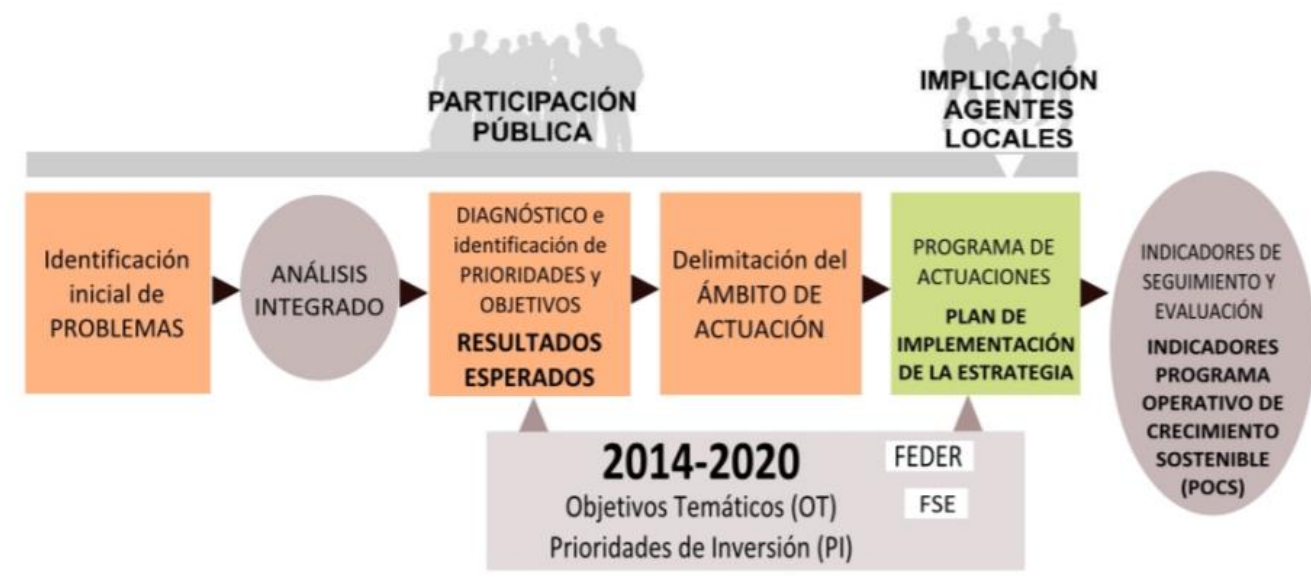

Fuente: Red de Iniciativas Urbanas (2015: 5)

Si bien no se cuenta aún con perspectiva temporal para poder valorar el impacto del programa EDUSI, lo que sí puede confirmarse es que este instrumento está contribuyendo a continuar la reflexión y la acción de las ciudades en relación a la regeneración de sus áreas en declive en el momento presente. Es de interés señalar que desde la Dirección General de Fondos Comunitarios, ente que gestiona a nivel nacional las EDUSI, se considera que en base a las mismas se ha dado "un cambio de $180^{\circ}$ en la planificación local de España" ${ }^{19}$, en tanto que el $100 \%$ de los municipios de al menos 50.000 habitantes ha presentado una estrategia a la primera convocatoria y las estrategias propuestas se consideran de "mucha calidad". En relación a esto, será clave el análisis de las mismas desde un enfoque crítico en el medio plazo, focalizando entre otras cuestiones en cómo han sido elaboradas dichas estrategias para entender si son el resultado de la capacidad administrativa de los propios municipios o de cosultoría externa.

Por otra parte, cabe afirmar que más allá del contexto de la programación de los Fondos Estructurales y de Inversión Europeo y de la Política de Cohesión, el Gobierno de España no ha desplegado una política nacional de desarrollo local, razón por la que

\footnotetext{
19 Afirmación recogida en una presentación de un representante del Ministerio de Hacienda y Administraciones Públicas durante el VI Pleno de la RIU, celebrado los días 3-4 de noviembre de 2016.
} 


\section{WPSReview International on Sustainable \\ Housing and Urban Renewal (RI-SHUR)}

también en este último periodo la dimensión urbana de la política de la UE vuelve a aparecer como un elemento clave de transformación del escenario de la regeneración urbana en el país. Además, hay que considerar que su capacidad de influir el marco nacional aumentará respecto a instrumentos anteriores, ya que el incremento de la financiación comunitaria permitirá que se desarrollen más de 150 estrategias EDUSI, una cifra muy por encima del número de programas financiados por URBAN, URBAN II, o la Iniciativa URBANA. A esto se une una intención de potenciar el papel que viene desarrollando la Red de Iniciativas Urbanas, entre otras cosas reforzando su capacidad de difusión y de intercambio de buenas prácticas entre las ciudades.

Para completar este marco, hay que considerar que en el momento presente el Gobierno de España ha iniciado el proceso de construcción de una Agenda Urbana para el país, en base a lo comprometido por España frente a la UE en el Acuerdo de Asociación de España 2014-2020. (Ministerio de Hacienda y Función Pública, 2014). Tras un periodo inicial en el que la tarea de la preparación de dicha Agenda se situó en el Ministerio de Hacienda y Función Pública, en la primavera de 2017 esta responsabilidad fue asumida por el Ministerio de Fomento que en la actualidad ha formado un Grupo de Expertos ${ }^{20}$ que está trabajando en un primer documento que previsiblemente saldrá a participación pública en la primavera de 2018. Este instrumento y el "gesto" político que conlleva tienen el potencial de generar una reflexión sobre el tema de la "Ciudad" que venga a sustituir la falta de visibilidad de este tema en el escenario político de nuestro país. El hecho de que una acción tan significativa se haya dado como resultado de un compromiso del Gobierno de España frente a la UE permite reafirmar la capacidad de influencia de la política comunitaria en el caso español, situando al país como un caso interesante en el marco de lo que se ha venido denominando Europeización de las políticas nacionales (González y Fedeli, 2015).

\section{Conclusiones}

El análisis realizado ha puesto de relieve la capacidad de la política comunitaria de influir en el marco nacional de la regeneración urbana a lo largo de todo el periodo de estudio. Esta circunstancia ha tenido lugar aun cuando la UE no tiene competencias

\footnotetext{
20 https://www.fomento.gob.es/MFOMBPrensa/Noticias/Fomento-impulsa-la-elaboraci\%C3\%B3n-de-laAgenda-Urbana/a370d7a2-a47d-4176-a346-2b1fc3309877
}

WPS RI-SHUR, n6, 2017, vol.2, ISSN: 2387-1768

MONOGRÁFICO SOBRE LAS ESTRATEGIAS DE DESARROLLO URBANO SOSTENIBLE E INTEGRADO (EDUSI) 


\title{
WPSReview International on Sustainable
}

\author{
Housing and Urban Renewal (RI-SHUR)
}

en relación a este tema, y ha sido propiciada, sobre todo, por la puesta a disposición de los EM de fondos para ayudar a las ciudades a frenar los procesos de degradación de sus áreas urbanas vulnerables. En este sentido, España ha mostrado un comportamiento muy parecido al de otros EM que no se habían dotado de una política urbana de ámbito nacional, si no fuera porque en el periodo 2007-2013 se desmarca del comportamiento de la mayor parte de los países miembros de la UE al lanzar con el apoyo del FEDER (artículo 8) un instrumento específico de regeneración, la Iniciativa URBANA, orientado explícitamente a dar continuidad a la Iniciativa Comunitaria URBAN. Ello se explica, en gran medida, por la atención política que el tema urbano recibió por parte del Gobierno en ese momento. El desarrolló de la Iniciativa URBANA permitió que un gran número de ciudades (46) siguieran diseñando e implementando estrategias integradas de regeneración y que el tema continuara contando con visibilidad y con la implicación de un número importante de actores institucionales y no institucionales que participaron en los programas.

La experiencia ganada en ese periodo emerge como clave para explicar la "facilidad" con la que desde nuestro país la Dirección General de Fondo Comunitarios del Ministerio de Hacienda y Administración Públicas pudo dar respuesta a la obligatoriedad impuesta desde la UE de dedicar al menos el $5 \%$ de los recursos del FEDER a financiar estrategias de desarrollo urbano sostenible integrado (EDUSI) en el periodo 2014-2020. Su diseño y su gestión están basados en gran medida en la experiencia obtenida en el periodo precedente, cuestión que sitúa a las EDUSI como claras continuadoras del enfoque URBAN. En efecto, aun teniendo en cuenta los cambios que las EDUSI han introducido en su convocatoria respecto a la Iniciativa URBANA (mencionados más arriba), están propiciando una continuación de la práctica de la regeneración urbana en línea con los principios de desarrollo urbano sostenible auspiciados por la UE. Esta circunstancia, que emerge al comparar las EDUSI con los instrumentos que las han precedido, está también presente en el discurso asumido por los principales actores institucionales que están trabajando en su implementación.

Tanto las EDUSI como el conjunto de elementos de transformación que la política urbana de la UE habría ido introduciendo en el país, o dicho de otro modo, la acomodación de España a la política de desarrollo urbano promovida por la UE, permiten afirmar que en el país se está avanzando hacia un mayor interés en torno al tema de la regeneración urbana. Aun así, la continuidad en la implementación del "método URBAN" sigue contrastando con la falta de una política urbana a nivel nacional acordada entre los tres niveles de gobierno (Gobierno de España, CCAA, y Ayuntamientos). Una política que, a pesar del interés que en ciertos momentos ha mostrado el Gobierno hacia el tema de la "Ciudad", nunca ha llegado a cristalizar en

WPS RI-SHUR, n6, 2017, vol.2, ISSN: 2387-1768

MONOGRÁFICO SOBRE LAS ESTRATEGIAS DE DESARROLLO URBANO SOSTENIBLE E INTEGRADO (EDUSI) 


\title{
WPSReview International on Sustainable
}

\author{
Housing and Urban Renewal (RI-SHUR)
}

un marco específico ${ }^{21}$. Esta circunstancia, podría explicase en gran medida por las reducidas competencias del Gobierno en relación al tema urbano, a diferencia de otros países de la UE menos descentralizados o que, contando con un grado de descentralización relevante, sí han conseguido dotarse de marcos comunes sobre el tema urbano a nivel nacional. Nuestro marco se caracteriza por el protagonismo que ocupan en la agenda política nacional las tensiones territoriales entre los diferentes niveles de gobierno (sobre todo entre CC.AA. y Gobierno), cuestión que dificulta el avance hacia escenarios consensuados en campos de competencias complementarias o concurrentes como el que nos ocupa. La recentralización que ha supuesto la aprobación de la Ley $27 / 2013$, de 27 de diciembre, de racionalización y sostenibilidad de la Administración Local (BOE, núm. 312, de 30 de diciembre), no ha implicado una mejora de esta situación.

La observación de los últimos acontecimientos, a la luz del devenir anterior, parece mostrar un posible cambio de rumbo hacia un marco consensuado en relación a la actuación en la ciudad existente, y en general en relación al desarrollo urbano sostenible. En efecto, en los últimos meses la "cuestión urbana" parece estar haciéndose un hueco en la agenda política nacional. En el mes de junio de 2017, el Ministro de Fomento, Íñigo de la Serna, comunicó el impulso del proceso de elaboración de la Agenda Urbana española. Como se ha explicado, su origen no se sitúa en una iniciativa de "cuño nacional" sino que se encuentra entre los compromisos adquiridos en el Acuerdo de Asociación de España 2014-2020, al que se suma el interés que el tema está ganando también en otros foros supranacionales ${ }^{22}$. Todo ello nos permite afirmar que los principales cambios que, en materia urbana, se están produciendo en nuestro país están fuertemente influenciados por la Política de Cohesión de la UE, y que los mismos podrían estar dando paso a cambios importantes en relación al tema que nos ocupa si son capaces de desplegar todo su potencial.

\section{Referencias bibliográficas}

Atkinson, R. y C. Rossignolo (2009). "An 'explicit' EU urban policy alter a 'learning' phase?". Ponencia presentada en la II European Urban Research Association Conference, Madrid (3-5 junio).

\footnotetext{
${ }^{21}$ Desde un punto de vista sustantivo se puede decir que el desarrollo urbano ha estado dominado por interpretaciones provenientes fundamentalmente de los ámbitos del urbanismo y la vivienda. Desde un punto de vista procedimental, ha estado condicionado por una Administración municipal "escuálida", alejada, en gran medida, de las exigencias y la sofisticación del tipo de gestión que requieren problemas cada vez más complejos.

${ }^{22}$ Por ejemplo, la implementación de la Agenda 2030 de Naciones Unidas con la que España se ha comprometido.
}

WPS RI-SHUR, n6, 2017, vol.2, ISSN: 2387-1768

MONOGRÁFICO SOBRE LAS ESTRATEGIAS DE DESARROLLO URBANO

SOSTENIBLE E INTEGRADO (EDUSI) 


\title{
WPSReview International on Sustainable
}

\author{
Housing and Urban Renewal (RI-SHUR)
}

Campagna, L. (2002). "Un bilancio del primo programma Urban, orientamento per i programmi futuri”. En PASQUI, G. y VALSECCHI, E. (coord.), II programma Urban e l'innovazione delle politiche urbane, Apprendere dall'esperienza: pratiche, riflessioni, suggerimenti (pp. 9-12). Milán: Terso Quaderno.

Carpenter, J. (2013). "Sustainable Urban Regeneration within the European Union. A case of 'Europeanization'?". En LEARY E.M. y J. McCARTHY: The Routledge Companion to Urban Regeneration, Londres: Routledge.

Carpenter, J. (2006). "Addressing Europe's Urban Challenges: Lessons from the EU URBAN Community Initiative". Urban Studies, 43(12), 2145-2162.

Chorianopoulos, I. (2002). "Urban Restructuring and Governance: North-South Differences in Europe and the EU URBAN Initiative", Urban Studies, 39(4), 705-726.

Comisión Europea (1994). "Notice to the Member States laying down guidelines for operational programmes which Member States are invited to establish in the framework of a community initiative concerning urban areas (URBAN)". DOCE no. L 180/37, de 01-07-1994, 06-09

Comisión Europea (2000). "Communication from the European Commission to the Member States laying down guidelines for a Community Initiative concerning economic and social regeneration of cities and of neighborhoods in crisis in order to promote sustainable urban development (URBAN II)". Bruselas, Publicaciones de la Comisión Europea.

Comision Europea (2003). "Partnership with the cities. The URBAN Community Initiative". Bruselas, Publicaciones de la Comisión Europea

Comisión Europea (2011). "Cities of tomorrow. Challenges, visions, ways forward", Informe de Expertos Externos, Bruselas.

Comisión Europea (2015). "Orientaciones para los Estados miembros Desarrollo sostenible integrado en el medio urbano Reglamento del FEDER (artículo 7)". Disponible en: http://ec.europa.eu/regional policy/es/information/publications/guidelines/2015/guidanc e-for-member-states-on-integrated-sustainable-urban-development-article-7-erdfregulation (último acceso 10-01-2018).

De Gregorio Hurtado, S. (2009). "El desarrollo de las Iniciativas Comunitarias URBAN y URBAN II en las periferias degradadas de las ciudades españolas", Ciudades, 13, 39-59.

De Gregorio Hurtado, S. (2012a). "Políticas urbanas de la Unión Europea desde la perspectiva de la Planificación Colaborativa. Las Iniciativas Comunitarias URBAN y URBAN II en España”. Tesis Doctoral. Departamento de Urbanística y Ordenación

WPS RI-SHUR, n6, 2017, vol.2, ISSN: 2387-1768

MONOGRÁFICO SOBRE LAS ESTRATEGIAS DE DESARROLLO URBANO

SOSTENIBLE E INTEGRADO (EDUSI) 


\section{WPSReview International on Sustainable \\ Housing and Urban Renewal (RI-SHUR)}

del Territorio, Escuela Técnica Superior de Arquitectura, Universidad Politécnica de Madrid.

De Gregorio Hurtado, S. (2012b). "La regeneración urbana en la acción estatal en España durante el periodo 2004-2011. Una historia a continuar". Perspectivas, 1, 2751.

De Gregorio Hurtado, S. (coord.), Dobson, S., Wyckmans, A., Coelho, D. (2013). "Urban regeneration as driver of adaptive capacity of cities". En IFLA Europe Journal, $2,40-47$.

De Gregorio Hurtado, S. (2014). "La Iniciativa Comunitaria URBAN como factor de transformación de la práctica de la regeneración urbana: aproximación al caso español", Ciudad y Territorio, 180, 253-275.

De Gregorio Hurtado, S. (2015). "Una reflexión crítica sobre la transposición de la política urbana de la Unión Europea al marco español". Cuadernos de Investigación Urbanística, 100, 19-23.

De Gregorio Hurtado, S. (2017a). "Is EU Urban Policy transforming Urban Regeneration in Spain? Answers from an analysis of the Iniciativa URBANA (20072013)". Cities, 60, 402-414.

De Gregorio Hurtado, S. (2017b). "La Política Urbana de la Unión Europea en España: de URBAN a las EDUSI", TRIA -Territorio della Ricerca su Insediamenti e Ambiente-, $18,47-74$.

Del Castillo, J. and S.N. Haarich (2013). "Desarrollo Urbano Sostenible cofinanciado por el FEDER en España 2014-2020: Directrices Estratégicas y Prioridades de Inversión", final Report for the DG Regio.

González Medina, M. (2011). "Elementos de una política urbana europea. Experiencias de gestión del desarrollo urbano en Alemania y España". Revista Española de Ciencia Política, 27, 45-67.

González Medina, M. (2013). "La europeización urbana a través de la Política de Cohesión". Revista CIDOB d'Afers Internacionals, 104, 133-154.

González Medina, M. y V. Fedeli (2015). "Exploring European Urban policy: Towards an EU-national urban agenda?", Gestión y Análisis de Políticas Públicas, 14 juliodiciembre.

Gutiérrez Palomero, A. (2009). La Unió Europea i la regeneració de barris amb dificultats. Tesis Doctoral. Departament de Geografia i Sociologia, Universitat de Lleida.

WPS RI-SHUR, n6, 2017, vol.2, ISSN: 2387-1768

MONOGRÁFICO SOBRE LAS ESTRATEGIAS DE DESARROLLO URBANO

SOSTENIBLE E INTEGRADO (EDUSI) 


\title{
WPSReview International on Sustainable
}

\author{
Housing and Urban Renewal (RI-SHUR)
}

Gutiérrez Palomero, A. (2010). "La Iniciativa Comunitaria URBAN y la construcción inconclusa de una Política urbana para la Unión Europea", Papeles de Geografía, 51$52,159-167$

Huete García, M.A.; Merinero Rodríguez, R. y Muñoz, R. (2016). “Urban regeneration policy from the Model of Integrated Urban Development in the European Union: an analytical approach based on the study of Spanish cities". Local Government Studies, 42(2), 267-286.

Instituto Universitario de Urbanística (2010). "Regeneración urbana integrada en Europa. Documento de Síntesis". Universidad de Valladolid, Disponible en: https://www.fomento.gob.es/nr/rdonlyres/94c72eb1-d0e7-428a-9039-

a73588c47866/95964/urban_regene_spanish.pdf (último acceso 10-12-2017).

Ministerio de Economía y Hacienda (2007a). Marco Estratégico Nacional de Referencia 2007-2013. Madrid: MEH

Ministerio de Economía y Hacienda (2007b). "Iniciativa URBANA (URBAN) Orientaciones para la elaboración de propuestas". Madrid: MEH

Ministerio de Hacienda y Función Pública (2014). Acuerdo de Asociación de España 2014-2020. Madrid: Ministerio de Hacienda y Función Pública.

Ministerio de Medio Ambiente y Medio Rural y Marino (2007a). Estrategia española de medio ambiente urbano (Borrador de noviembre de 2007). Madrid: MAMRM

Ministerio de Medio Ambiente y Medios Rural y Marino (2007b). Libro verde de medio ambiente urbano. Tomo I. Madrid. MAMRM.

Ministerio de Medio Ambiente y Medio Rural y Marino [MAMRM] (2011). Estrategia española de sostenibilidad urbana y local. Madrid: MAMRM.

Ministerio de Obras Públicas Transporte y Medioambiente (1996). Primer Catálogo Español de Buenas Prácticas. I Conferencia ONU Asentamientos Humanos. Madrid: MOPTMA.

Ministerio de la Presidencia (2007). Estrategia Española de Desarrollo Sostenible. Madrid. Ministerio de la Presidencia.

Parkinson, M. (2005). "Urban Policy in Europe. Where have we been and where are we going?". Documento para el NODE Project on European Metropolitan Governance for Austrian Federal Ministry of Education, Science \& Culture.

Red de Iniciativas Urbanas (2015). "Orientaciones para la definición de Estrategias de Desarrollo Urbano Sostenible Integrado en el periodo 2014-2020". Disponible en: http://www.rediniciativasurbanas.es/NR/rdonlyres/EFED876C-9E2C-4C89-9880-

WPS RI-SHUR, n6, 2017, vol.2, ISSN: 2387-1768

MONOGRÁFICO SOBRE LAS ESTRATEGIAS DE DESARROLLO URBANO

SOSTENIBLE E INTEGRADO (EDUSI) 
WPSReview International on Sustainable

Housing and Urban Renewal (RI-SHUR)

C17D7D3BACB2/135389/1 20150803 OrientacionesEstrategiasIntegradas20149.pdf (último acceso 10-01-2018).

Reunión Informal de ministros de desarrollo urbano de la UE (2007). Leipzig Charter. Leipzig.

Disponible

en:

http://ec.europa.eu/regional_policy/archive/themes/urban/leipzig_charter.pdf (último acceso 10-01-2018).

Reunión Informal de ministros de desarrollo urbano de la UE (2010). Declaración de Toledo.

Disponible

en:

http://ec.europa.eu/regional_policy/archive/newsroom/pdf/201006_toledo_declaration_ en.pdf (último acceso 10-01-2018).

Tofarides, M. (2003). Urban Policy in the European Union: A multi-level gatekeeper system. Ashgate: Aldershot/Burlington.

WPS RI-SHUR, n6, 2017, vol.2, ISSN: 2387-1768

MONOGRÁFICO SOBRE LAS ESTRATEGIAS DE DESARROLLO URBANO

SOSTENIBLE E INTEGRADO (EDUSI) 